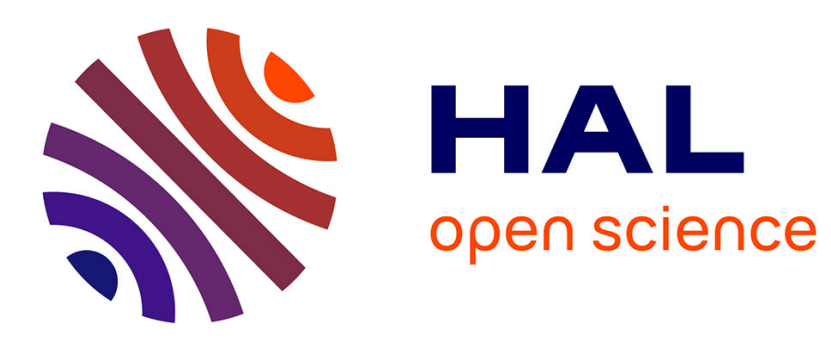

\title{
Etude d'un système de pompe à chaleur thermochimique pour le stockage de l'énergie et la fourniture de chaleur et de froid
}

\author{
S. Elberg, P. Mathonnet
}

\section{- To cite this version:}

S. Elberg, P. Mathonnet. Etude d'un système de pompe à chaleur thermochimique pour le stockage de l'énergie et la fourniture de chaleur et de froid. Revue de Physique Appliquée, 1982, 17 (9), pp.585-590. 10.1051/rphysap:01982001709058500 . jpa-00245035

\section{HAL Id: jpa-00245035 https://hal.science/jpa-00245035}

Submitted on 1 Jan 1982

HAL is a multi-disciplinary open access archive for the deposit and dissemination of scientific research documents, whether they are published or not. The documents may come from teaching and research institutions in France or abroad, or from public or private research centers.
L'archive ouverte pluridisciplinaire HAL, est destinée au dépôt et à la diffusion de documents scientifiques de niveau recherche, publiés ou non, émanant des établissements d'enseignement et de recherche français ou étrangers, des laboratoires publics ou privés. 
Classification

Physics Abstracts

$86.30 \mathrm{R}-86.40 \mathrm{H}$

\title{
Etude d'un système de pompe à chaleur thermochimique pour le stockage de l'énergie et la fourniture de chaleur et de froid
}

\author{
S. Elberg et P. Mathonnet \\ C.E.A., Centre d'Etudes Nucléaires de Grenoble, Département des Réacteurs à Eau, \\ Service des Transferts Thermiques, 85X, 38041 Grenoble cedex, France
}

(Reçu le 21 septembre 1981, accepté le 28 décembre 1981)

\begin{abstract}
Résumé. - Le présent article concerne une étude visant à prouver la faisabilité d'un système de pompe à chaleur thermochimique capable de stocker l'énergie et de la restituer sous forme de chaleur et de froid. Après l'exposé du principe de fonctionnement du système, un dispositif de mise en ouvre est décrit et les tests expérimentaux ayant permis de vérifier ses performances sont présentés. Les résultats d'une étude économique simple sont ensuite donnés.
\end{abstract}

\begin{abstract}
The paper concerns a study intended to prove the feasibility of a thermochemical heat pump system able to store energy and restore it under heat and cold. After explanation of the working principle, an experimental configuration is described and results of some tests are presented. A simple economic study is then given.
\end{abstract}

Introduction. - Le stockage de la chaleur sous forme d'énergie chimique, qui consiste à effectuer à volonté les réactions exothermique et endothermique inverses d'un équilibre chimique présente par rapport aux autres moyens de stockage thermique des avantages marquants (forte capacité de stockage, délais de restitution indifférent) [1], [2]. Il permet, de plus, d'obtenir un effet de pompe à chaleur mis à profit dans des systèmes actuellement désignés sous le nom de pompes à chaleur thermochimiques.

Il nous a paru intéressant de rassembler ces deux propriétés dans un dispositif de stockage capable de restituer l'énergie à la fois sous forme de chaleur et de froid.

Un tel dispositif permettrait d'adapter la production de capteurs solaire ou toute autre source de chaleur peu coûteuse mais intermittente, aux besoins simultanés en chaleur et en froid de certaines industries, en particulier, de l'industrie agro-alimentaire pour ses processus de stérilisation, pasteurisation, séchage, évaporation, etc.

Nous nous proposons de rapporter ici les principaux résultats d'une étude visant à prouver la faisabilité d'un tel système. Après avoir exposé son principe de fonctionnement, nous présenterons le dispositif que nous avons retenu pour sa mise en œuvre et les résultats de tests expérimentaux ayant permis de vérifier les performances de ce dernier. Nous donnerons ensuite les résultats d'une étude économique simple.
1. Principe de fonctionnement. - 1.1 EquILIBRE CHOISI. - L'utilisation d'un équilibre de décomposition thermique d'ammoniacates de sels métalliques du type :

$$
\begin{array}{r}
\text { A, } m \mathrm{NH}_{3} \text { (solide) } \rightleftarrows \mathrm{A}, n \mathrm{NH}_{3} \text { (solide) }+ \\
+(m-n) \mathrm{NH}_{3} \text { (gaz) }
\end{array}
$$

endothermique dans le sens décomposition et exothermique dans le sens absorption nous paraît offrir un certain nombre d'avantages :

- Les réactions sont réversibles et fiables, rappelons de ce point de vue que certaines d'entre elles ont été utilisées sans aucun problème pendant des années dans des armoires frigorifiques ménagères commercialisées entre les deux guerres,

- Les quantités de chaleur mises en jeu sont notables du fait de la forte énergie d'interaction entre le moment dipolaire de la molécule d'ammoniac et les ions des sels métalliques,

- L'ammoniac est un bon fluide frigorifique (forte chaleur de vaporisation, tension de vapeur élevée au voisinage de la température ambiante), les produits solides utilisés sont pour la plupart stables et peu coûteux.

1.2 Fonctionnement. - Le fonctionnement du système à l'aide d'un tel équilibre est schématisé sur la figure 1 . Il correspond à un cycle intermittent de pompe à chaleur à absorption. 


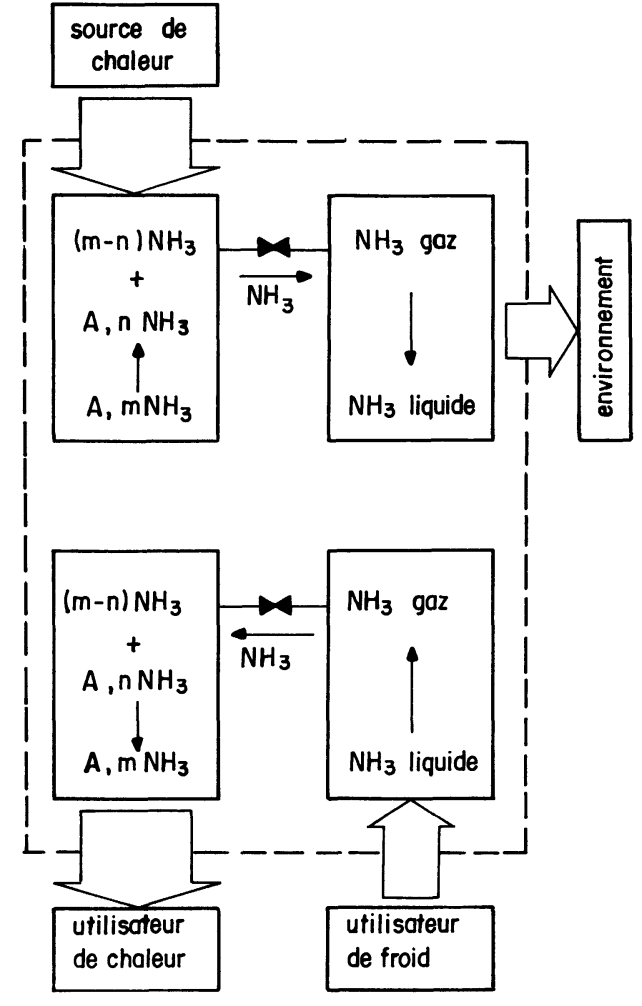

Fig. 1. - Principe de fonctionnement du système.

[Working principle.]

L'équilibre est mis en ouvre dans deux appareils identiques constitués de deux enceintes étanches communiquant par l'intermédiaire d'une vanne et équipées chacune d'un échangeur.

Chaque appareil est alternativement soumis à une phase de charge et à une phase de décharge :

- pendant la charge, il emprunte à une source de chaleur la chaleur de décomposition de l'ammoniacate A, $m \mathrm{NH}_{3}$ et rejette vers l'extérieur la chaleur de condensation de l'ammoniac,

- pendant la décharge, il emprunte à l'extérieur la chaleur de vaporisation de l'ammoniac et fournit à un utilisateur la chaleur de formation de l'ammoniacate $\mathrm{A}, m \mathrm{NH}_{3}$.

$\mathrm{Au}$ cours de cette dernière phase, l'appareil est donc capable de fournir de la chaleur et du froid.

L'équilibre étant monovariant, la température de condensation et d'évaporation de l'ammoniac fixe les conditions de fonctionnement de chaque appareil pendant les deux phases : pression, température au-dessus de laquelle la chaleur doit être fournie et au-dessous de laquelle elle peut être récupérée.

Dans le cas de l'utilisation de l'équilibre de décomposition thermique de l'hexaammoniacate de chlorure de nickel :

$$
\mathrm{NiCl}_{2}, 6 \mathrm{NH}_{3} \rightleftarrows \mathrm{NiCl}_{2}, 2 \mathrm{NH}_{3}+4 \mathrm{NH}_{3}
$$

ces conditions de fonctionnement sont données sur la figure 2. Elles sont compatibles avec la production

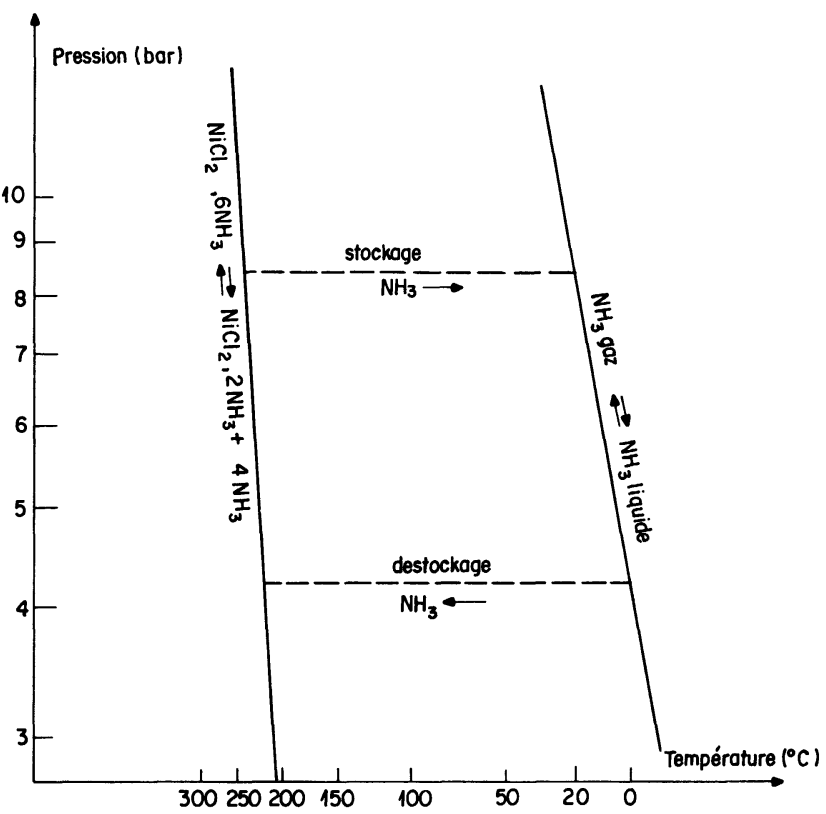

Fig. 2. - Conditions de fonctionnement théoriques du système $\mathrm{NiCl}_{2} / \mathrm{NH}_{3}$.

[Theoretical working conditions of the $\mathrm{NiCl}_{2} / \mathrm{NH}_{3}$ system.]

d'eau glacée ct de vapeur à $180^{\circ} \mathrm{C}$ et 10 bars à partir des capteurs solaires pseudo-paraboliques ou à miroirs segmentés actuellement développés (température de sortie : $\left.300^{\circ} \mathrm{C}\right)$ :

- pour une température de condensation de l'ammoniac de $20^{\circ} \mathrm{C}$, la pression pendant la charge est de 8,55 bars et la chaleur doit être fournie à une température supérieure à $242^{\circ} \mathrm{C}$,

- pour une température d'évaporation de l'ammoniac de $0^{\circ} \mathrm{C}$, la pression pendant la décharge est de 4,30 bars et la chaleur peut être récupérée à une température inférieure à $219{ }^{\circ} \mathrm{C}$.

Le rapport de la puissance thermique extraite à $0^{\circ} \mathrm{C}$ à la puissance thermique fournie à $219^{\circ} \mathrm{C}$ est de 0,37. L'utilisation de l'équilibre de décomposition de l'hexaammoniacate de chlorure de nickel suivant un tel cycle de pompe à chaleur à absorption procure un grand écart entre la température à laquelle la chaleur est pompée et la température à laquelle elle est rejetée, ce qui permet de valoriser à la fois la chaleur pompée et la chaleur rejetée. L'énergie nécessaire au fonctionnement du cycle est alors utilisée avec une efficacité globale supérieure à 1 .

L'intermittence du cycle procure un moyen de stockage intéressant et d'une grande souplesse d'utilisation :

- La capacité de stockage par unité de masse des produits mis en réserve $(56 \%$ sous forme de chlorure de nickel et $44 \%$ sous forme d'ammoniac) est d'environ $296 \mathrm{Wh}$ d'énergie thermique délivrée à haute température. Dans la même gamme de température $\left(180-300^{\circ} \mathrm{C}\right)$, la chaleur sensible de l'eau 
sous pression (10-80 bars) ne fournirait qu'une capacité de stockage voisine de $160 \mathrm{Wh} / \mathrm{kg}$, la chaleur sensible d'un fluide thermique organique à pression atmosphérique une capacité de stockage voisine de $80 \mathrm{Wh} / \mathrm{kg}$.

- L'effet de stockage est obtenu par la simple fermeture de la vanne séparant les deux enceintes; la régulation du débit d'ammoniac permet une régulation fine des puissances thermiques.

D'autres équilibres peuvent être utilisés, en particulier les équilibres de décomposition de l'hexaammoniacate de chlorure de magnésium :

$$
\mathrm{MgCl}_{2}, 6 \mathrm{NH}_{3} \rightleftarrows \mathrm{MgCl}_{2}, 2 \mathrm{NH}_{3}+4 \mathrm{NH}_{3}
$$

et de l'hexaammoniacate de chlorure de manganèse :

$$
\mathrm{MnCl}_{2}, 6 \mathrm{NH}_{3} \rightleftarrows \mathrm{MnCl}_{2}, 2 \mathrm{NH}_{3}+4 \mathrm{NH}_{3} \text {. }
$$

Leurs conditions de fonctionnement et leurs performances sont données dans le tableau I.

Tableau I. - Conditions de fonctionnement et performances de divers équilibres.

[Working conditions and performances of different

\begin{tabular}{|c|c|c|c|c|}
\hline & \multirow{2}{*}{\multicolumn{3}{|c|}{ Equilibres }} \\
\hline & & & & \\
\hline & & $\mathrm{NiCl}_{2} / \mathrm{NH}_{3}$ & $\mathrm{MgCl}_{2} / \mathrm{NH}_{3}$ & $\mathrm{MnCl}_{2} / \mathrm{NH}_{3}$ \\
\hline \multirow{3}{*}{ 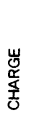 } & Température $\mathrm{NH}_{3}\left({ }^{\circ} \mathrm{C}\right)$ & \multicolumn{3}{|c|}{20} \\
\hline & Pression (bar) & \multicolumn{3}{|c|}{8,55} \\
\hline & Température poudre $\left({ }^{\circ} \mathrm{C}\right)$ & 242 & 205 & 157 \\
\hline \multirow{3}{*}{  } & Température $\mathrm{NH}_{3}\left({ }^{\circ} \mathrm{C}\right)$ & \multicolumn{3}{|c|}{0} \\
\hline & Pression (bar) & \multicolumn{3}{|c|}{4,30} \\
\hline & Tempërature poudre $\left({ }^{\circ} \mathrm{C}\right)$ & 219 & 179 & 134 \\
\hline \multicolumn{2}{|c|}{ Capacıté de stockage (Wh/kg) } & 296 & 275 & 207 \\
\hline \multicolumn{2}{|c|}{ Composition massique $(\%)$} & $56 / 44$ & $48 / 52$ & $55 / 45$ \\
\hline \multicolumn{2}{|c|}{$\frac{\text { Puissance extraite (froid) }}{\text { Puissance fournie (chaud) }}$} & 0,37 & 0,47 & 0,54 \\
\hline
\end{tabular}
equilibriums.]

2. Mise en æuvre. -2.1 CONCEPTION DU DISPOSITIF. - La mise en œuvre du système soulève un certain nombre de problèmes au premier rang desquels il faut signaler :

- la faible conductivité thermique des lits de poudre,

- la forte influence de la température de ces lits sur la cinétique des réactions,

- la variation importante de volume qui accompagne l'absorption d'ammoniac.

$\mathrm{Au}$ sein des lits de poudre d'ammoniacate, les transferts de chaleur se font principalement par conduction gazeuse dans les espaces laissés libres par les grains. La conductivité thermique est donc faible, de l'ordre de 1 à $2 \mathrm{~mW} / \mathrm{cm}^{\circ} \mathrm{C}$ et dépend de la densité apparente du lit.

La cinétique des réactions de décomposition et d'absorption semble obéir à une loi du premier ordre dáns le cas des ammoniacates, la constante de vitesse étant une fonction pratiquement linéaire de l'écart de température et de pression par rapport à l'équilibre. Pour une pression de condensation ou d'évaporation de l'ammoniac donnée, la vitesse de ces réactions ne pourra donc être augmentée que par variation de température des lits de poudre.

En théorie, lors du passage du chlorure pur à l'hexoammoniacate, le volume du lit de poudre est respectivement multiplié par 4,2, 3,5 et 4,5 dans le cas du chlorure de nickel, de magnésium et de manganèse.

Un dispositif capable de mettre en œuvre le système dans les meilleures conditions possibles doit donc satisfaire à deux conditions essentielles :

- les poudres doivent y être réparties en lits de faible épaisseur,

- la variation de volume de ces lits doit être prévue afin que le compactage des lits qu'elle entraîne ne bloque pas les transferts de matière.

La configuration que nous avons retenue est schématisée sur la figure 3 . Le lit de poudre est réparti autour d'un tube à ailettes à l'intérieur duquel circule le fluide caloporteur, la poudre étant maintenue en place à l'aide d'une enveloppe perméable.

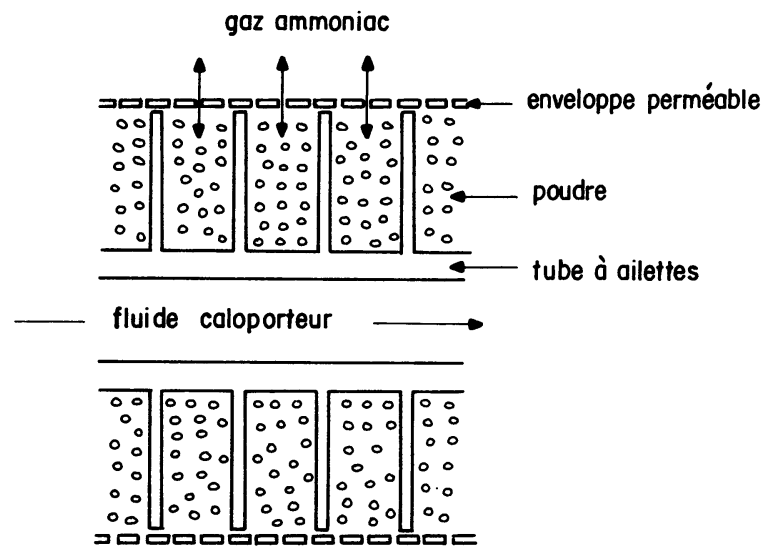

Fig. 3. - Dispositif pour la mise en œuvre du système. [Experimental configuration.]

Une telle configuration procure, à notre avis, de nombreux avantages :

- le lit de poudre est fractionné en compartiments de faibles dimensions, ce qui favorise les échanges de matière et de chaleur,

- dans ces compartiments, la poudre conserve une certaine fluidité et une certaine homogénéité vis-à-vis des variations de volume,

- les compartiments sont clos ce qui empêche la poudre d'être repoussée loin de la surface d'échange,

- l'échangeur de chaleur offre une grande surface d'échange,

- le lit de poudre baigne totalement dans l'ammoniac gazeux, 
- le remplissage peut aisément être effectué en prévoyant une partie amovible dans l'enveloppe; la faible dimension des compartiments permet d'obtenir des taux de remplissage initiaux faibles ce qui, après absorption d'ammoniac, laisse un lit suffisamment poreux pour ne pas trop gêner la circulation de l'ammoniac,

- l'ensemble forme un module facilement réalisable à partir de matériels courants,

- un grand nombre de ces modules peuvent être regroupés, en série ou en parallèle, sous forme de faisceaux compacts.

2.2 Tests eXPéRimentauX. - Ces tests avaient pour but de vérifier les performances du dispositif dans les conditions de fonctionnement du système. Ils ont été effectués sur un banc d'essais du laboratoire, à l'aide d'un module expérimental déjà utilisé pour une étude de stockage thermique [3].

L'échangeur est un tube à ailettes en acier inoxydable du commerce. Les ailettes carrées de $5 \mathrm{~cm}$ de côté sont serties sur un tube de 20/23. L'espacement entre ailettes est de $4 \mathrm{~mm}$ et les ailettes ont une épaisseur de $0,5 \mathrm{~mm}$. La longueur du tube est de $55 \mathrm{~cm}$ ce qui procure une surface d'échange de $0,65 \mathrm{~m}^{2}$ pour un volume utilisable de 11 . L'enveloppe perméable est constituée par un panier en tôle perforée doublé d'une toile d'acier inoxydable. La partie supérieure du module peut être ouverte pour le remplissage ou l'observation des lits de poudre en cours de réaction. Deux thermocouples permettent de mesurer la température de la poudre.

La charge du module correspond à 3,6 moles de $\mathrm{NiCl}_{2}$. Au cours des 15 cycles de stockage-déstockage qu'elle a subis pour la précédente étude, en couplage avec un second lit d'ammoniacate, nous avons pu constater :

- que le lit n'est soumis à une variation de volume importante que lors de la première absorption d'ammoniac; les grains de chlorure de nickel anhydre se brisent en une poudre fine qui se compacte et forme un bloc rigide adhérant aux parois des ailettes,

- que lors des réactions ultérieures, les propriétés de ce lit ainsi compacté sont bien reproductibles; le compactage semblant laisser un lit de poudre suffisamment poreux pour ne pas gêner la circulation de l'ammoniac.

Les résultats des essais de déstockage dans le cadre de la présente étude sont représentés sur la figure 4. Cette figure donne, en fonction du temps, les variations de la quantité d'ammoniac absorbée et de la température du lit de poudre pour trois réactions d'absorption successives effectuées dans des conditions identiques (température initiale de la poudre : $180^{\circ} \mathrm{C}$, pression d'ammoniac : 9 bars).

Ces essais mettent une nouvelle fois en évidence la reproductibilité des propriétés du lit de poudre.

L'allure des courbes est caractéristique d'une réaction dont la cinétique est limitée par les échanges
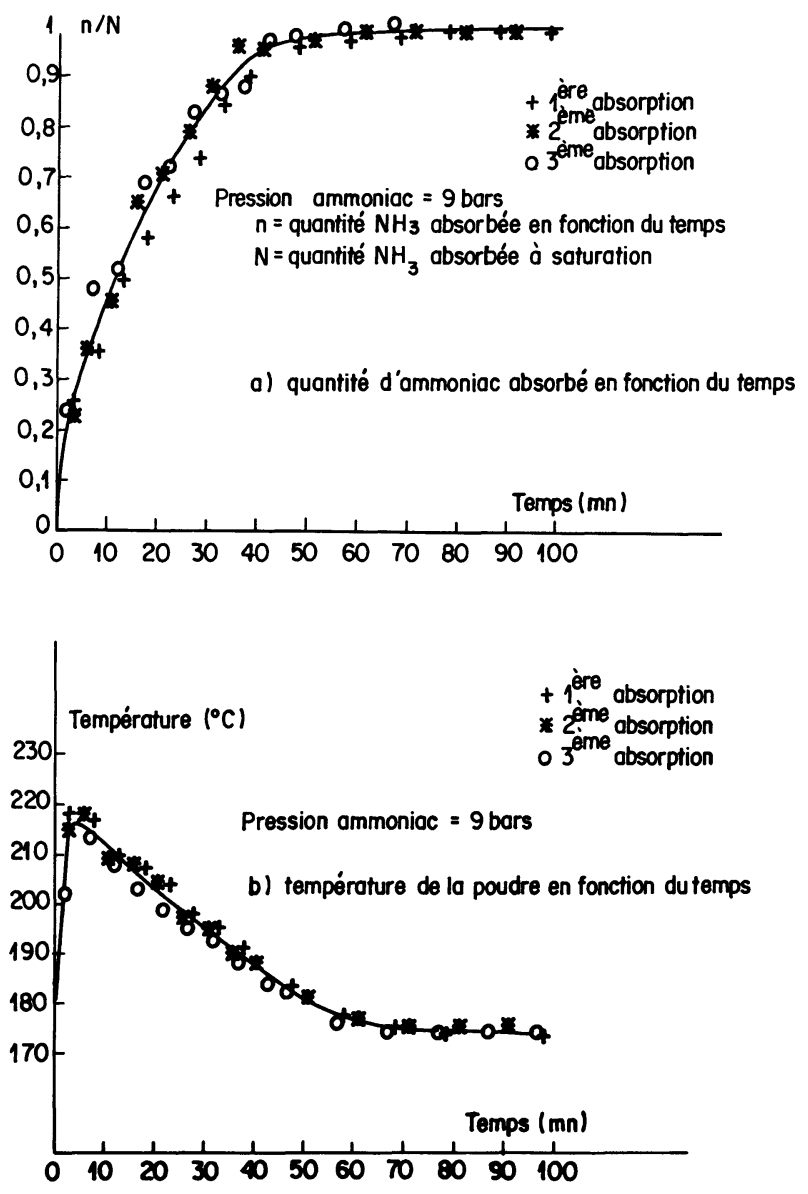

Fig. 4. - Essais de déstockage.

[Discharging tests.]

de chaleur. Dans les premiers instants, l'absorption de l'ammoniac se traduit par une élévation brusque de la température de la poudre. La réaction d'absorption devient alors très lente et doit attendre le refroidissement de la poudre pour se poursuivre.

Au bout de $40 \mathrm{~min}, \mathbf{9 5} \%$ de la quantité théorique d'ammoniac est absorbée. Le débit d'ammoniac est alors en moyenne de $4,80 \mathrm{~mole} / \mathrm{h}$ par mole de chlorure de nickel ce qui correspond à une puissance thermique théorique de $297 \mathrm{~W}$ libérée au sein de la charge de chlorure de nickel.

Au cours de la même période, la puissance recueillie à l'aide du fluide caloporteur circulant à l'intérieur du tube à ailettes est en moyenne de $164 \mathrm{~W}$, soit $55 \%$ de la puissance théorique.

Les pertes thermiques s'expliquent principalement par les difficultées que nous avons rencontrées pour mettre en place un calorifugeage efficace du module expérimental; elles devraient être réduites de façon appréciable pour un module de plus grande taille.

Ces pertes s'expliquent aussi par le fait qu'une partie de la chaleur dégagée par la réaction sert à élever la température du gaz ammoniac de la température ambiante à la température de la réaction. Pour une élévation de température de $160^{\circ} \mathrm{C}$, cette 
fraction de chaleur correspond à $11 \%$ de la puissance thermique théorique.

Ces essais nous ont permis d'autre part de calculer un coefficient global d'échange de $11 \mathrm{~W} / \mathrm{m}^{2}{ }^{\circ} \mathrm{C}$, valeur en bon accord avec les valeurs de 7 à $14 \mathrm{~W} / \mathrm{m}^{2}{ }^{\circ} \mathrm{C}$ publiées dans le cas d'autres ammoniacates [4].

Les caractéristiques du banc d'essais (température $\max 300^{\circ} \mathrm{C}$, pression max 10 bars) nous ont obligés à effectuer les essais de stockage dans des conditions où les réactions de décomposition sont lentes $(90 \%$ de la quantité théorique d'ammoniac libérée en $160 \mathrm{~min}$.). Ces essais se sont également avérés bien reproductibles.

Afin de tester la souplesse de régulation du système, nous avons soumis le module expérimental à une suite de créneaux de pression. La figure 5 donne, en fonction du temps, les variations de la pression d'ammoniac et de la quantité de chaleur à fournir au système pour le maintenir à une température constante.

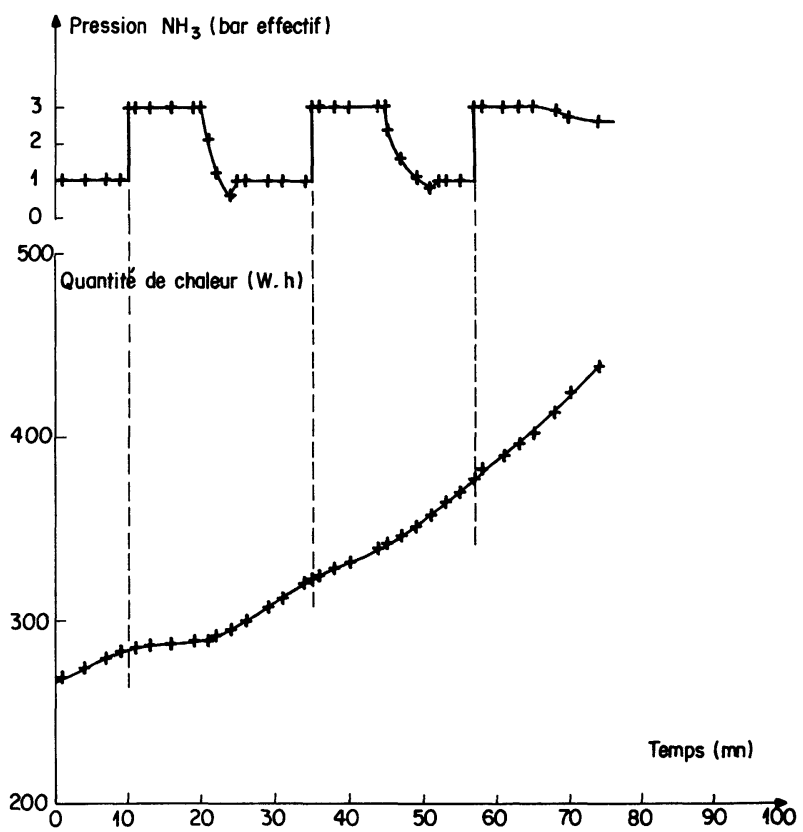

Fig. 5. - Essais de régulation.

[Regulation tests.]

On peut alors constater que la " réponse thermique " du système aux deux premiers créneaux est relativement bonne. Elle devient plus floue par la suite du fait du ralentissement de la réaction d'absorption.

3. Données économiques - Afin de fixer l'ordre de grandeur des investissements que nécessite un tel système, nous avons déterminé ses caractéristiques dans le cas de la satisfaction des besoins d'un utilisateur appelant une puissance thermique de $1 \mathrm{~kW}$ et une puissance frigorifique de $0,37 \mathrm{~kW}$ pendant $8 \mathrm{~h} /$ jour $(2400 \mathrm{~h} / \mathrm{an})$ à l'aide de capteurs solaires à hautes températures (rendement 0,50 ) fonctionnant $5 \mathrm{~h} /$ jour $(1800 \mathrm{~h} / \mathrm{an})$ avec un ensoleillement moyen de $800 \mathrm{~W} / \mathrm{m}^{2}$.

Le stockage a été dimensionné de façon à assurer une autonomie de $8 \mathrm{~h}$.

Pour le système, nous avons retenu les hypothèses suivantes :

- capacité de stockage de $213 \mathrm{Wh} / \mathrm{kg}$ pour tenir compte d'un rendement matière de 0,90 et d'un rendement thermique de 0,80 ,

- coefficient d'échange thermique de $10 \mathrm{~W} / \mathrm{m}^{2}{ }^{\circ} \mathrm{C}$ côté poudre et $500 \mathrm{~W} / \mathrm{m}^{2}{ }^{\circ} \mathrm{C}$ côté ammoniac liquide,

— densité des produits : $1 \mathrm{~kg} / 1$ pour l'ammoniacate de chlorure de nickel dans son état de saturation maximum et $0,61 \mathrm{~kg} / 1$ pour l'ammoniac liquide.

En prenant un écart de température de $30^{\circ} \mathrm{C}$ côté poudre et de $5^{\circ} \mathrm{C}$ côté ammoniac liquide et un volume des échangeurs de $5 \mathrm{l} / \mathrm{m}^{2}$, les caractéristiques techniques et économiques du système sont données sur la figure 6 et dans le tableau II où elles sont comparées à celles d'un système hybride dans lequel les besoins thermiques en l'absence de soleil sont assurés par une chaudière au fuel (rendement 0,90 ) et les besoins frigorifiques fournis par une machine à absorption (rendement 0,70 ).

Le système que nous proposons nécessite un investissement légèrement supérieur à celui du système hybride concurrent, ses frais de fonctionnement sont, par contre, plus faibles (pas de consommation de fuel et moindre consommation d'eau de refroidissement), ses frais d'entretien semblent également plus faibles du fait que le dispositif est statique et scellé.

Il faut noter la faible part des investissements propres au système $(25 \%)$ dans l'investissement total; la rentabilité du système devrait donc être meilleure dans le cas de l'utilisation de sources d'énergie intermittentes nécessitant un investissement plus faible que les capteurs solaires, le courant d'heures creuses par exemple.

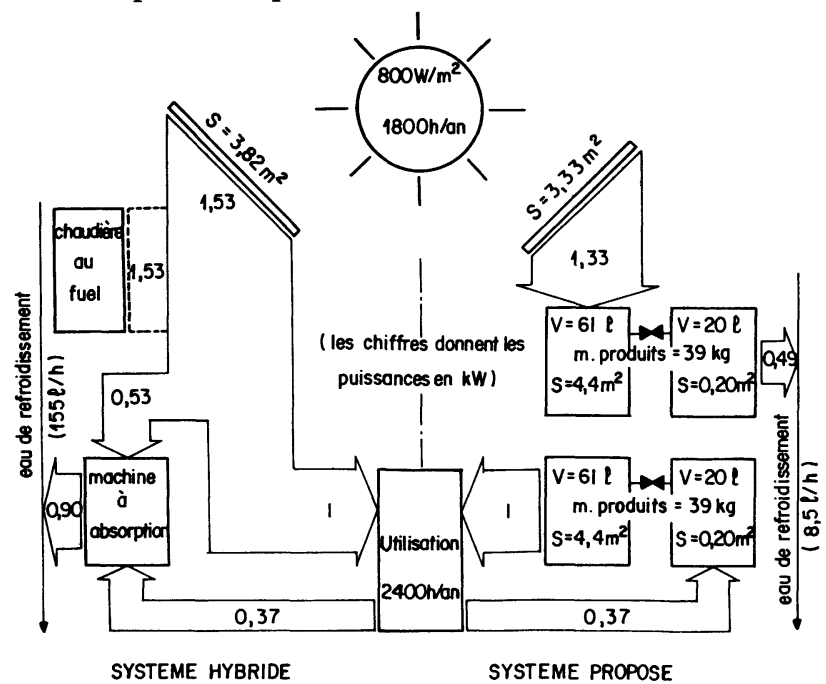

Fig. 6. - Caractéristiques techniques du système proposé.

[Technical characteristics of the proposed system.] 
Tableau II. - Caractéristiques économiques du système proposé.

[Economical characteristics of the proposed system.]

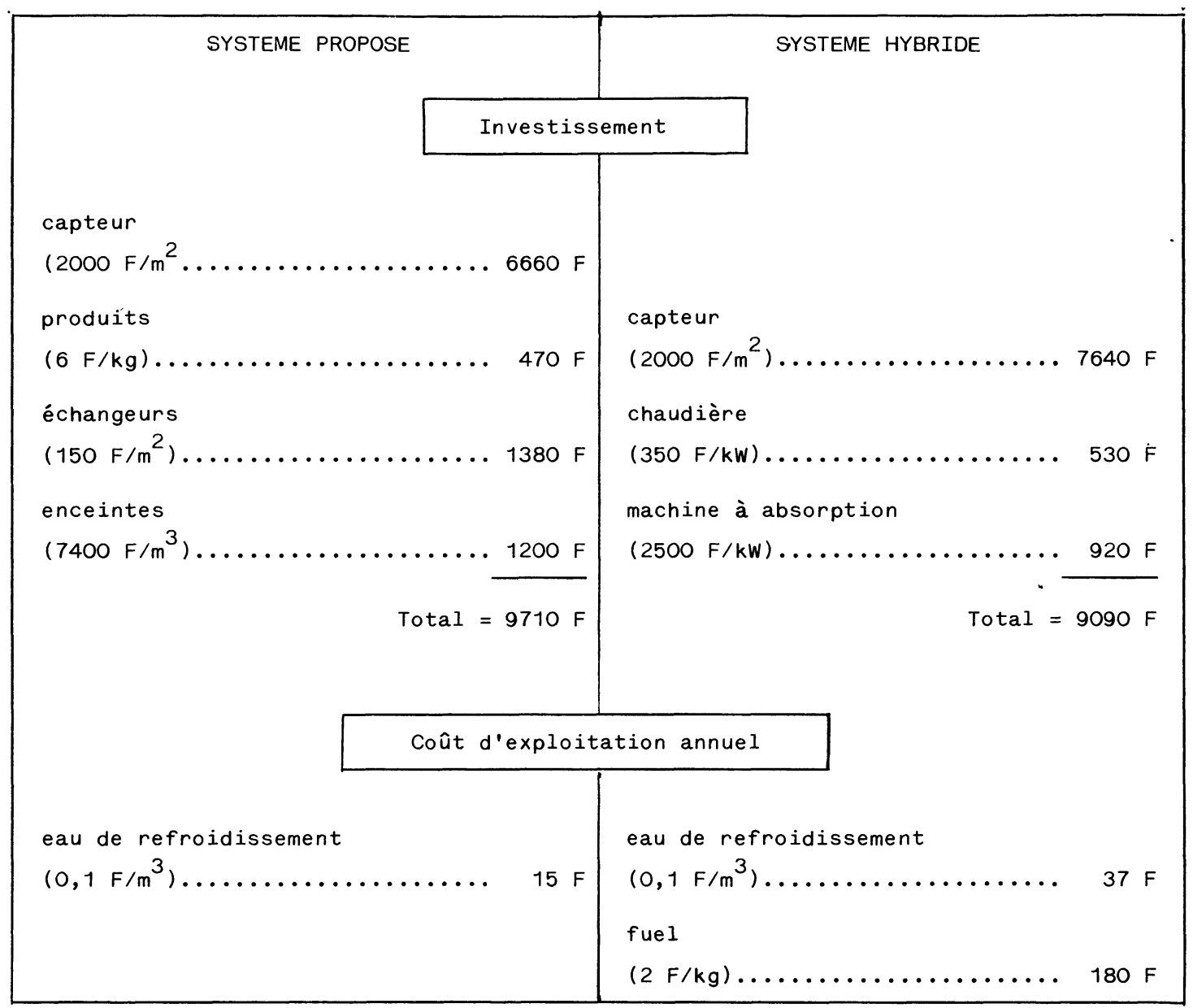

4. Conclusion. - La présente étude de faisabilité ne constitue qu'une première étape; elle a permis de mettre en évidence :

- la bonne reproductibilité des performances d'un module expérimental,

- de situer les conditions réelles de fonctionnement de ce module,

— de fixer l'ordre de grandeur de son coût.
Elle se poursuit actuellement par la construction et l'étude du fonctionnement d'un prototype d'une dizaine de $\mathrm{kWh}$ équipé d'un échangeur à ailettes en aluminium. Des mesures plus fines, la prise en compte des effets de taille, une plus grande précision dans l'évaluation des investissements devraient ainsi, à notre avis, permettre de prouver la faisabilité du système à l'échelle industrielle.

\section{Bibliographie}

[1] Elberg, S., Mathonnet, P., «Principales réactions dissociatives étudiées pour le stockage chimique de l'énergie thermique. Problèmes de mise en œuvre ». Colloque "Le Génie Chimique et le Stockage de l'Energie ", Paris les 8 et 9 décembre 1980.

[2] Elberg, S., MathonNEt, P., « Le stockage de la chaleur sous forme d'énergie chimique à moyennes et basses températures". Colloque "Le Stockage de l'Energie Solaire Appliqué au Bâtiment. Néces- sité et Avenir pour le Solaire ", Lyon les 21 et 22 janvier 1981.

[3] Contrat COMES no 79-0149. "Stockage de la chaleur entre 130 et $200^{\circ} \mathrm{C}$ sous forme d'énergie chimique ». Rapport à paraître.

[4] Howerton, M. T., "A thermochemical energy storage system and heat pump. Proc. Int. Energy Conversion Eng. Conf., San Diego (août 1978) p. 935 . 